\title{
Setting up a heart emergency centre
}

\author{
SJS Virk, E Edmunds, RA Perry
}

\begin{abstract}
Summary
We describe the setting up of a 'heart emergency centre' linked to a pre-existing coronary care unit. The 'heart emergency centre' offers a new and efficient way of providing a fast and effective system for the assessment and management of patients with cardiac emergencies. This set-up can result in significant improvements in 'door to needle' time for thrombolysis, which has been shown to be beneficial both in terms of morbidity and mortality following myocardial infarction.
\end{abstract}

Keywords: cardiac centre, emergency centre

\begin{tabular}{|l|}
\hline Steering Committee \\
\hline - Chief Executive \\
- Consultant Cardiologists (2) \\
- Consultant Anaesthetist \\
- Director of Nursing \\
- local General Practitioners (2) \\
- Consultant Physicians (2) \\
- Director of Finance \\
- Information Manager \\
- Ambulance Service \\
representatives (2) \\
Senior Coronary Care Sister \\
\hline
\end{tabular}

Box 1

The Cardiothoracic Centre, Thomas Drive, Liverpool L14 3PE, UK SJS Virk

E Edmunds

RA Perry

Correspondence to Dr SJS Virk, Warrington Hospital, Lovely Lane, Warrington WA5 1QG, UK

Accepted 10 February 1997
A rapid and effective response to myocardial infarction has been targeted as a major health gain area by the Department of Health in the UK, ${ }^{1}$ and health districts have been recommended to draw up policies to ensure that patients with suspected infarction gain rapid access to facilities for resuscitation and thrombolysis. In their guidelines for the early management of patients with myocardial infarction, the British Heart Foundation Working Group, reporting in the $B M F$ in March $1994,{ }^{2}$ concluded that, depending on local circumstances, achieving the desired standards may involve direct admission to coronary care units.

Direct-access chest pain emergency centres is a concept which is already being widely adopted in the US. ${ }^{3}$ In August 1994 a rapid access assessment centre for cardiothoracic emergencies was opened at the Cardiothoracic Centre in Liverpool. Here we describe the establishment of the Heart Emergency Centre (HEC) and report on its first 12 months.

\section{Background}

The Cardiothoracic Centre in Liverpool is a tertiary centre which provides a comprehensive service in cardiothoracic surgery and investigative and interventional cardiology for Merseyside, North Wales, Cheshire, Isle of Man and parts of Lancashire, with a combined population of approximately three million people.

The Cardiothoracic Centre also provides coronary care facilities for Broadgreen District Hospital with which it shares a site in East Liverpool. The catchment population for Broadgreen Hospital, which serves the South and East of Liverpool, totals approximately 280000 . Patients admitted to the Accident and Emergency department (A\&E) of Broadgreen Hospital with cardiothoracic emergencies, eg, myocardial infarctions, thoracic aortic dissections and unstable angina, were referred to the Cardiothoracic Centre following initial assessment in A\&E. As the A\&E department of Broadgreen Hospital was remote from the coronary care unit and thrombolysis was not given there, the 'door to needle' time for thrombolysis was often unacceptably long (average $111 \mathrm{~min}$ ). As several major trials have shown that early thrombolysis reduces mortality and improves outcome following myocardial infarction ${ }^{4-6}$ a new system for dealing with these emergencies was needed.

In 1994, because of a trust merger, the emergency services were rearranged so that the A\&E facilities would be concentrated at the Royal Liverpool Hospital some two miles away. A phased closure of the Broadgreen A\&E department commenced in August 1994. It was felt that the resulting reduction in access to emergency cardiac services would be detrimental to the population of South and East Liverpool. This provided the impetus for the development of the Heart Emergency Centre (HEC).

\section{Method}

Three months prior to the opening of the HEC a steering committee was set up to discuss the aims and requirements of the service (box 1). Representatives of the steering committee also held regular meetings with paramedic groups and local general practitioners (GPs) so that they were updated regarding the plans and could contribute ideas. The agenda for the steering committee could be divided into four main sub-headings, site/accommodation, equipment, personnel and referral source/pattern.

\section{SITE ACCOMMODATION}

Ease of access was an important factor in determining the location of the new unit. The HEC, based at the Cardiothoracic Centre Liverpool, consists of two previously unoccupied rooms in a six-bedded coronary care unit. The coronary care unit is situated on the ground floor next to the main entrance 


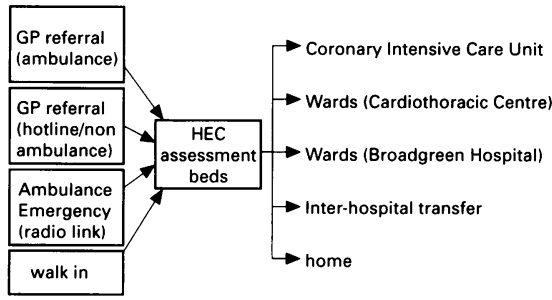

Figure 1 Service model outline

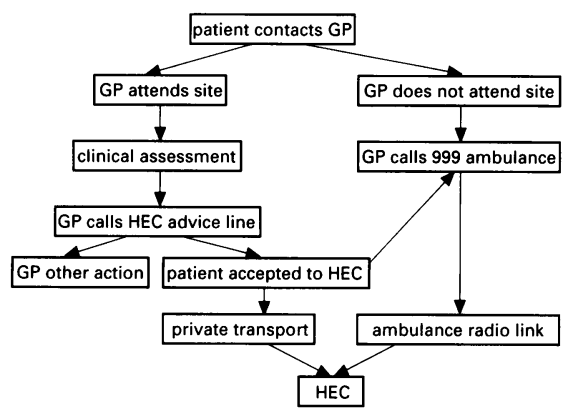

Figure 2 GP referral protocol

\begin{tabular}{|l|}
\hline $\begin{array}{l}\text { Summary of paramedic } \\
\text { criteria for HEC referral }\end{array}$ \\
\hline - suspected acute myocardial \\
infarction \\
- suspected other cardiovascular \\
emergency, eg, dissecting aortic \\
aneurysm, acute pericarditis, \\
pulmonary embolus, pulmonary \\
infarct \\
- acute illness of uncertain cause \\
with past history of heart disease \\
- acute illness or cardiovascular \\
emergency of any type in previous \\
Cardiothoracic Centre patient
\end{tabular}

Box 2 and adjacent to the ambulance bay. Both rooms are large and no structural changes were necessary to adapt them for their present use. As these rooms were situated in the coronary care unit, thrombolysis could be started immediately under the supervision of highly trained staff, thus eliminating the time taken to transfer patients from the $A \& E$ department.

\section{EQUIPMENT}

\section{Monitoring}

Both HEC rooms are equipped with monitors featuring 12-lead ST segment analysis and electrocardiogram printing facilities.

\section{Resuscitation}

The two rooms are equipped with full resuscitation facilities including defibrillators and external pacemakers.

\section{Communications link}

A two-way radio link was installed at the nursing station, enabling paramedic crews to warn the HEC staff of imminent arrivals. A direct telephone link with ambulance control was also installed.

\section{PERSONNEL}

\section{Medical}

The coronary care unit has a resident Senior House Officer whose duties included the assessment and management of referrals from the A\&E department. As a doctor is constantly present on the coronary care unit the opening of the HEC has reduced the time taken for patients to be assessed. There is also Registrar and Consultant level input available at all times.

\section{Nursing staff}

In order to maintain the coronary care nurse : patient ratio of $1: 2$, the setting up of the two additional HEC beds necessitated the employment of seven additional full time nursing staff.

\section{Clerical staff}

The added workload in terms of data collection, filling of routine clinical results and informing general practitioners of all HEC visits within 24 hours of patient consultation, required the employment of one additional full-time ward clerk.

REFERRAL PROTOCOL

Following discussions between Liverpool Health Authority, the Cardiothoracic Centre and the Mersey Regional Ambulance Service, an outline service model was developed for the HEC (figure 1). The aim was to establish a 'fast track' in the early management of cardiovascular emergencies, in particular the rapid diagnosis of acute myocardial infarction with the positive goal of reducing 'door to needle' time for thrombolytic treatment. Prior to the opening of the HEC most of the referrals to the coronary care unit had occurred via the $A \& E$ department at Broadgreen Hospital.

\section{General practitioners}

The new protocol allowed GPs to refer patients with suspected cardiothoracic emergencies directly to the HEC. This could be done in a number of different ways and is outlined in figure 2 .

\section{Paramedic ambulance}

When the HEC service was being planned, the Mersey Regional Training Manager and Area Manager for Paramedic Services were co-opted onto the steering committee. This led to the development of precise guidelines to enable patients with cardiac emergencies to be referred directly to the HEC by paramedic crews. A quick reference summary of the ambulance admission protocol is given in box 2. Paramedic crews are encouraged to inform the HEC of referrals via the radio link prior to their arrival.

\section{Self-referral and others}

Patients who are known to the Cardiothoracic Centre can telephone the HEC for advice which may lead to admission. Occasionally patients may be referred to the HEC via other hospital departments. 


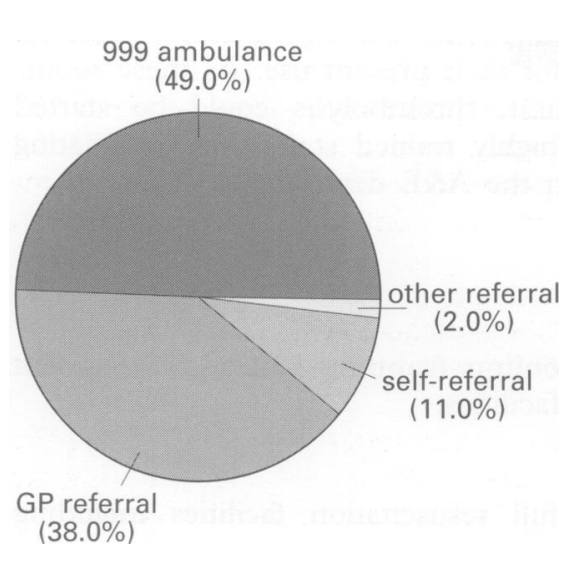

Figure 3 Source of referral $(n=1695)$

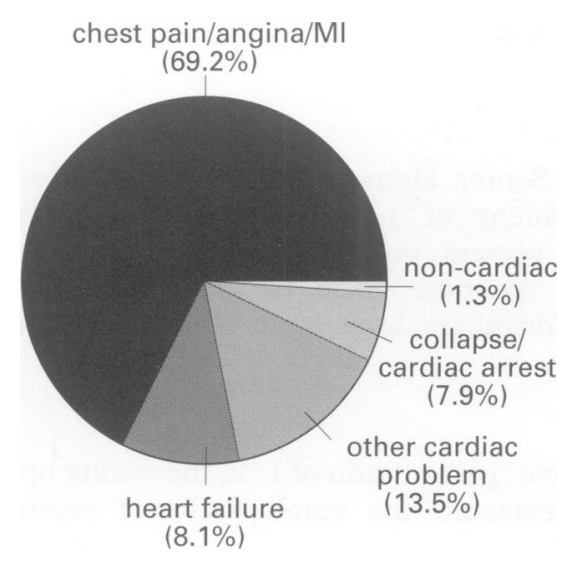

Figure 4 Provisional diagnosis/reason for referral $(n=1695)$

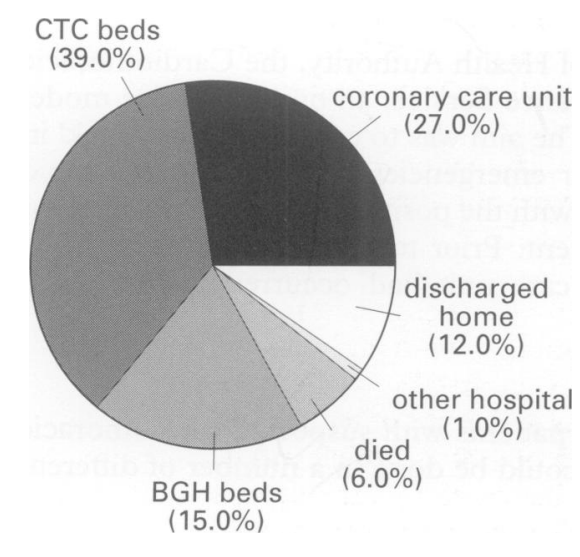

Figure 5 Discharge destination $(n=1695)$

Table Thrombolysis data

\begin{tabular}{lll}
\hline & Pre-HEC & Post-HEC \\
\hline $\begin{array}{c}\text { Average 'door to } \\
\text { needle' time (min) }\end{array}$ & 111 & 38 \\
$\begin{array}{c}\text { Patients receiving } \\
\text { thrombolysis }\end{array}$ & 46 & 90.7 \\
within 90 min (\%) & & \\
\hline
\end{tabular}

\section{Results}

During its first year of operation 1695 patients attended the HEC, giving an average daily attendance of four to five patients. A statistical breakdown for the first 12 months is given below.

\section{PATIENT DEMOGRAPHICS}

There were 1020 male patients (60.2\%), compared with 675 (39.8\%) females. The median age for female attendees was 68 years $(41-89$ years, 5th-95th centile), compared with 63 years $(38-83$ years, 5 th -95 th centile) for males.

\section{SOURCE OF REFERRAL}

Patient-initiated emergency 999 calls were responsible for 831 (49\%) of admissions, 644 (38\%) via GP referral, 186 (11\%) via self-referral and $34(2 \%)$ via other referral sources (figure 3 ).

\section{PROVISIONAL DIAGNOSIS/REASON FOR REFERRAL}

The most common reason for referral was chest pain or suspected myocardial infarction which occurred in 1173 patients (69.2\%) (figure 4). The other common reasons for referral were collapse/cardiac arrest and heart failure. The group categorised as 'other cardiac problems' numbered 229, and of these 124 (54.1\%) represented patients with suspected cardiac arrhythmia/palpitations.

\section{HEC DIAGNOSIS}

From the 1173 patients referred with chest pain to the HEC, $191(16.3 \%)$ had a documented myocardial infarction and 441 (37.6\%) were diagnosed as having unstable or stable angina pectoris. The remaining $541(46.1 \%)$ patients were classified as chest pain, ? cause, with the exception of eight $(0.7 \%)$ patients who had thoracic aortic aneurysms. Of the 134 patients admitted having had a collapse/cardiac arrest, 87 (64.9\%) had a documented cardiopulmonary arrest, whilst the rest were classified as collapse, ? cause, with the exception of two patients who had documented strokes.

\section{DISCHARGE DESTINATIONS}

Just over a quarter (458/1695) of the patients were admitted to the coronary care unit, while another 661 (39\%) were admitted to beds in the Cardiothoracic Centre and $254(15 \%)$ to beds at Broadgreen Hospital (figure 5). Only 102 $(6 \%)$ of the admissions to the HEC died whilst on the unit, while a further 17 (1\%) were transferred to other hospital units; 203 patients (12\%) were discharged home from the HEC.

\section{THROMBOLYSIS}

Of the 191 patients with a documented myocardial infarction, $107(56 \%)$ were eligible for thrombolytic therapy. Most of these patients $(90.7 \%)$ received thrombolysis within 90 minutes, this compared with only $46 \%$ of patients meeting this target prior to the setting up of the HEC. The average "door to needle' time also decreased from 111 minutes to 38 minutes following the opening of the HEC (table).

\section{Discussion}

The management of cardiac emergencies such as acute myocardial infarction has changed radically over the last decade. In particular, it has been shown that the early administration of intravenous thrombolytic agents substantially reduces early mortality after acute myocardial infarction by restoring blood flow in occluded coronary arteries. ${ }^{7}$ This has led to the re-evaluation of current hospital-based practices for the management of these patients. Increasingly emergency departments are organising units dedicated to the rapid triage and examination of patients with symptoms suggestive of ischaemic heart disease. ${ }^{3}$ Such efforts are intended to decrease the often unacceptably long delay between patient arrival in the emergency department and initiation of therapy. ${ }^{8}$

The HEC, as outlined in this article, provides one alternative for the delivery of an assessment and management service for cardiac emergencies. Our experience suggests that the HEC may provide a fast and effective triage system for the evaluation of these patients. Almost all of the patients attending the HEC had appropriate symptoms at the time of referral, ie, only 22 out of the 1695 patients seen had a provisional referral diagnosis which was thought to be non-cardiothoracic in nature (figure 2). The referrals to the HEC would also appear to be appropriate in that the vast majority of patients $(88 \%)$ required 
1 Secretary of State for Health. The health of the nation: a consultative document for health in England. London: HMSO, 1991; p 59.

2 Weston CFM, Penny WJ, Julian DG. Guidelines for the early management of patients with myocardial infarction. $B M \mathcal{F}$ 1994; 308: 767-71. 3 Shesser R, Smith $M$. The chest pain emergency department and the outpatient chest pain evaluation centre. Ann Emerg Med 1994; 23: 33441.

4 Gruppo Italiano per lo Studio della Streptochinasi nell'Infarcto Miocardico (GISSI). Effectiveness of intravenous thrombolytic treatment in acute myocardial infarction. Lancet 1986; $397-401$.

hospitalisation (figure 5), and only $12 \%$ of patients were deemed suitable for direct discharge from the HEC.

The majority of referrals (49\%) occurred via patient-initiated emergency 999 calls (figure 3). Despite this, our results would appear to indicate that most of these referrals to the HEC were appropriate in terms of patient symptoms (figure 2). This probably occurred as a result of the detailed guidelines provided to paramedical ambulance staff.

The opening of the HEC resulted in a major improvement in the 'door to needle' time for thrombolysis with $90.7 \%$ of patients having a 'door to needle' time of under 90 minutes and the average 'door to needle' time decreasing from 111 minutes to 38 minutes (table).

The efficient usage of HEC beds seen in our series probably reflects the extensive interaction that took place between the different disciplines during the planning stages, resulting in detailed guidelines for referral. As well as highlighting the importance of appropriate referrals, our series also demonstrates the importance of having sufficient District General Hospital beds for the onward referral of patients in order to maximise the use of beds in the HEC. In our series 254 of the 1695 patients (15\%) were admitted directly from the HEC to District General Hospital beds. This problem with onward referral of patients is one of the major concerns associated with the setting up of a HEC centre linked to a pre-existing coronary care unit.

5 Second International Study of Infarct Survival Collaborative Group. Randomised trial of intravenous streptokin. Cravenous strep 17,187 cases of suspect neither among 17,187 cases of suspected acute myocardial infarction: ISIS-2. Lancet 1988; ii:

6 GUSTO Investigators. An International randomised trial comparing four thrombolytic strategies for acute myocardial infarction. $N$ Engl $\mathcal{f}$ Med 1993; 329: 673-82.
7 Muller DW, Topol EJ. Selection of patients with acute myocardial infarction for thrombolytic therapy. Ann Intern Med 1990; 113: 949-60.

8 Sharkey SW, Brunette DD, Ruiz E, et al. An analysis of time delays preceding thrombolysis for acute myocardial infarction. $f A M A 1989$; for acute myoca $3171-4$

Michael Nicholls

President

The Fellowship of Postgraduate Medicine 